\title{
Detection of Street-Parking Vehicles Using Line Scan Camera and Scanning Laser Range Sensor
}

\author{
Kiyotaka HIRAHARA and Katsushi IKEUCHI \\ Ikeuchi Laboratory \\ Institute of Industrial Science, University of Tokyo \\ 4-6-1 Komaba, Meguro-ku, Tokyo, 153-8505, Japan \\ E-mail: hirahara@iis.u-tokyo.ac.jp
}

\begin{abstract}
Street-parking vehicles in urban areas are causing various traffic problems, however, have been counted manually in the traffic census, including counting error. We propose two auto-detection methods; one is based on an epipolar-plane image analysis, using a line-scan camera, and the other is based on three-dimensional restoration, using a scanning laser range sensor. Both methods achieve high detection rate. Furthermore, we introduce two-type panoramic street images, obtained by vertically line-scanning camera and laser sensors. Street-parking vehicles side-images will be extracted from the panoramic street images.
\end{abstract}

\section{Introduction}

In crowded urban areas, vehicles parked on streets occupy a certain area of the streets at any time and cause traffic problems, such as slow-moving traffic and blind spots on roads. It is very important for traffic census to obtain the number of those vehicles. Actual information about street-parking vehicles is useful for re-planning of roads and traffic system. At present traffic census, measurement of those actual conditions is performed manually. Especially, for actual traffic condition survey in Japan, street-parking vehicles have to be manually counted by investigators in measuring vehicles. Manual counting has some problems, for example, human error in counting and high cost.

We are interested in automatic measurement of the number of those vehicles. We have already proposed the detection method (1), which can detect street-parking vehicles by using a scanning laser range sensor. The laser range sensor is active and lets us know the distance to target objects directly. In this method, first we reconstructed three-dimensional model from acquired range data. Second, we extracted road surface and vehicles' side bodies. Third, we drew occluding boundary curve and silhouette edge curve, respectively. We applied thresholds to two curves and could count the number of vehicles. In the ideal measurement condition, the detection rate resulted in $100 \%$. However, when there are other objects between the laser range sensor and target vehicles, they create occlusion and target vehicles cannot be counted correctly. For example, some vehicles go past the measurement vehicle.

This paper describes also two alternative methods to detect street-parking vehicles. One is based on an epipolar-plane image (EPI) analysis, which are easily obtained by a line-scan camera. EPI analysis, firstly developed by Bolles ${ }^{(2)}$, is a technique for building a three-dimensional description of a static scene from a dense sequence of images. EPI analysis is used to calculate the features depth from the slope of feature paths in EPIs.

The other method is based on three-dimesional restoration, from range data obtained by a scanning laser range sensor. A series of processing at each scan is repeated, and counts how many clusters of range data indicating vehicles side appear.

We will introduce two-type panoramic street images; one is obtained by a line-scan camera, and the other is obtained by a scanning laser range sensor. It will be shown that both images are used simultaneously and can derive useful information for our everyday life.

\section{Detection Method Using Line Scan Camera}

\subsection{Epipolar-Plane Image Analysis}

In the same way of two-camera stereo, an epipolar-plane image is also obtained from a sequence of images when a camera's lens center moves in a straight line. When a camera moves in a straight line, pointed perpendicularly to its trajectory, the epipolar plane is the plane on which there are each point $P$ of the scene and the lens center's trajectory. This camera's motion is named a lateral motion. Epipolar lines on these image planes for a lateral motion are collinear and parallel to the lens-center's trajectory. 
These epipolar lines construct an epipolar-plane image, in the right side of Figure 1. A point $P$ of the scene, called (scene-) feature point, draws a path in the epipolar-plane image, referred as feature path. When a camera's velocity in its lateral motion is constant, all feature paths are straight in the epipolar-plane image.

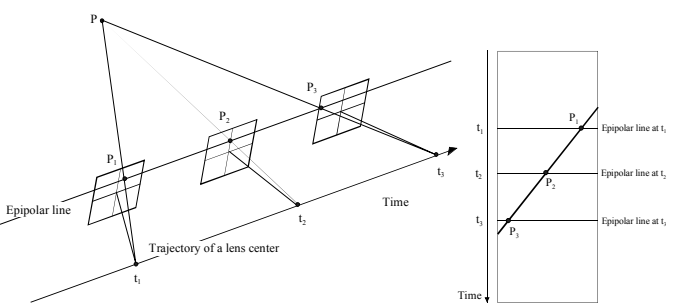

Figure 1 An epipolar-plane image for a camera's lateral motion: a camera's lateral motion and an epipolar line on its image plane at each time (left), the epipolar-plane image contained of a sequence of epipolar lines (right).

For a period $\Delta t=t_{2}-t_{1}$, the projected point of the feature point $P$ onto the image plane moves from $P_{1}$ to $P_{2}$, at a distance of $\Delta u$ between $P_{1}$ and $P_{2}$ in the image plane. The image plane and the feature point $P$ are at a distance $h$ and $D$ from the lens-center's trajectory respectively. It is assumed that the velocity $V$ of the lens center is constant. The proportion of $D$ to $h$ is same as the proportion of $V \times \Delta t$ to $\Delta u$, so that the relationship between the depth $D$ of the feature point $P$ from the camera's trajectory and the slope $m=\Delta t / \Delta u$ of the feature path in the epipolar-plane image is derived as the following equation,

$$
D=h \times \frac{V \times \Delta t}{\Delta u}=h \times V \times m
$$
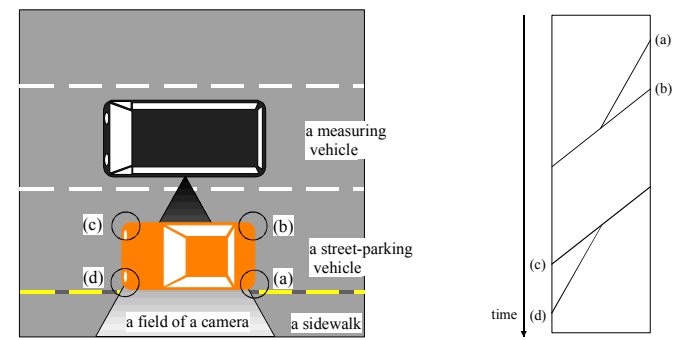

Figure 2 Measuring situation and expected EPI: each feature point draws the corresponding feature path in EPI

\subsection{Algorithm Using Line-Scan Camera}

(1) Image Split

The EPI is split by every several pixels in the direction of time, in order to find feature paths coming up in the EPI in chronological order. In this splitting process, there is no duplication and no gap occurring among all split small images.
(2) Edge Detection

So as to find edges in each split image, we use the Canny edge detector ${ }^{(3)}$, which finds edges by looking for local maxima of the gradient of each image. The gradient is calculated using the derivative of the Gaussian filter. The method uses two thresholds; the low threshold is 0.0125 and the high threshold is 0.0313. The standard deviation of the Gaussian filter $\sigma$ is 1 . Each split image results in a binary image with 1's where edges are found and 0's elsewhere by the Canny edge detector.

\section{(3) Line Extraction}

Straight lines lying concealed in each split binary image could be detected using the Hough transformation $^{(4)}$ which is implemented in the Radon function applied to the binary image. The locations of strong peaks in the Radon transform matrix correspond to the locations of straight lines in the original image. A straight line in each split image is represented by $x \cos \theta+y \sin \theta+\rho=0$, where the origin of the coordinate system is the center of the image, $\theta$ is the normal angle of this line with reference to the $\mathrm{x}$-axis and $\rho$ is the distance of this line from the origin. The range of $\theta$ is 1 to 180 degrees and the range of $\rho$ is within plus or minus a half length of a diagonal line of the split image. In the Hough transformation, quantization for an angle $\theta$ is at 1 degree.

The strongest peak is only made a search for and selected, and then the corresponding straight line could be regarded as the most remarkable feature path in each split binary image. Finally, the slope of the selected feature path means approximately the depth of the corresponding feature point from the lens center at the moment the corresponding image was taken.

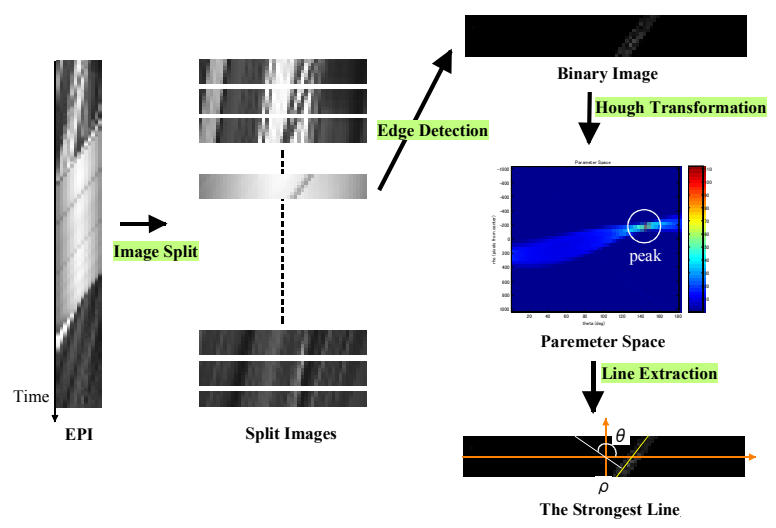

Figure 3 Image processing diagram

\subsection{Experiments}

After the image processing procedure mentioned 
above, the slope of feature paths in EPIs informs us the depth to the corresponding feature points in the scene. In this paper, we use the slope of the projective line from 1 to 180 degrees in stead of the depth between feature points and the lens center. However, calculation of depth from slope is important and it is described here.

For the slope of the feature path $m$, the distance $D$ between the corresponding feature point and the lens center of the camera can be calculated by the following equation.

$$
D=\frac{v \times m \times p}{2 \times f \times \tan \frac{\theta}{2}},
$$

where $v(\mathrm{~m} / \mathrm{s})$ is the velocity of the measuring vehicle, $m$ is the slope of the feature path, $p$ (pixels) is the number of pixels of a line scan camera, $f(\mathrm{~Hz})$ is the frequency of scanning a line, and $\theta$ (degrees) is the angle of field of the camera.

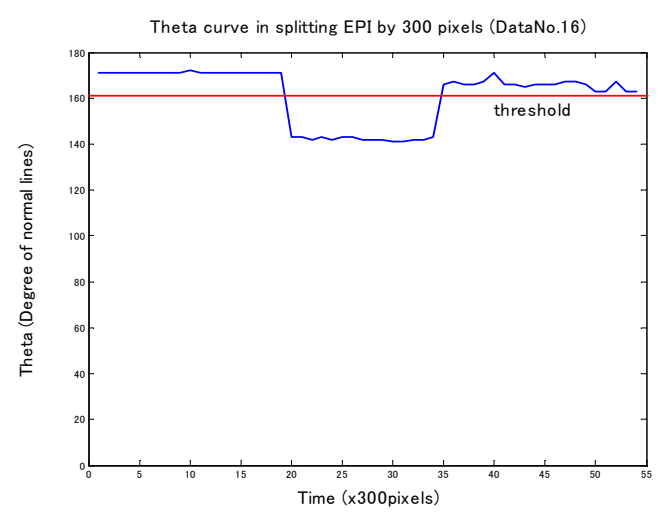

Figure 4 Slope of normal lines of the feature paths plotted against time with split size 300 pixels

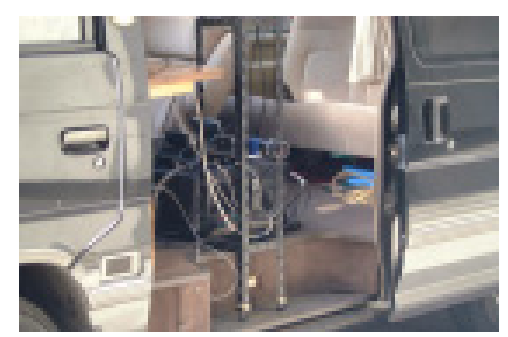
Figure 5 A line scan camera mounted on the
measuring vehicle

Our developed measuring vehicle measures vehicles parked on Route 246 in Tokyo, Japan. As a result of the measurement, an EPI is obtained by a line scan camera, DALSA SP-14-02k30. Figure 2 shows that the vehicle parked on the street appears as three light-gray belts in the middle of the image. The height of the camera is $900 \mathrm{~mm}$, the velocity of the probe vehicle is $2.78 \mathrm{~m} / \mathrm{s}(10 \mathrm{~km} / \mathrm{h})$, the number of pixels of a line scan camera is 2048 pixels, the frequency of scanning a line is $7 \mathrm{kHz}$, and the angle of field of the camera is 62 degrees.
After the image processing, Figure 4 provides a plot of the slope of the feature paths $m+90$ degree against time. There are the changes of the slope corresponding to the vehicle parked on the street in Figure 4. The distance $D$ between the side surface of the street-parking vehicle and the measuring vehicle is calculated to be 1 meter for $m=145-90=55$ degrees by the equation.

When the measuring vehicle goes straight at 4-level of velocities; $10 \mathrm{~km} / \mathrm{h}, 20 \mathrm{~km} / \mathrm{h}, 30 \mathrm{~km} / \mathrm{h}$ and $40 \mathrm{~km} / \mathrm{h}$, the rate of detection of street-parking vehicles is summarized in Table 1. In this summary, the estimated threshold values are used and the rate of detection is calculated as the number of vehicles detected by this system respect to the sum of the number of vehicles parked actually and the number of vehicles detected wrongly. Relationship between velocity of the measuring vehicle and the rate of detection could not be found. The entire rate of detection is $71 \%$.

\begin{tabular}{|c|c|}
\hline Speeed $(\mathrm{km} / \mathrm{h})$ & Rate $(\%)$ \\
\hline \hline 10 & 66 \\
\hline 20 & 100 \\
\hline 30 & 75 \\
\hline 40 & 60 \\
\hline
\end{tabular}

Table 1 Rate of detection with respect to 4-levels of velocities

Distortion occurs from the specular reflection on the side of target vehicles, the reflection of the surroundings onto the side surface of target vehicles, and the special structure of target vehicles. The specular reflection inputs a strong light beam into the lens, and then obtained images are over a range of a CCD's sensitivity. The reflection of the surroundings causes the remarkable feature paths in EPIs. In the image processing, they are detected wrongly. In results, wrong depth is calculated. Trucks have different body structure than common vehicles. Trailer and tractor could be detected separately to be two vehicles.

\section{Panoramic Street-Image}

\subsection{Line Scan Imaging}

Perhaps the most common example of line scan imaging is the fax machine. Line scan imaging uses a single line of sensor pixels (effectively one-dimensional) to build up a two-dimensional image. The second dimension results from the motion of the object being imaged. Two-dimensional images are acquired line by line by successive single-line scans while the object moves perpendicularly past the line of pixels in the image sensor.

The laser range sensor used in line scan imaging, realizes one more dimension. The third dimension results as the range data between the object and the 
detector. Three-dimensional images are also acquired line by line while the object moves perpendicularly past the scanning line.

Line scan imaging has some benefits, including:

- Dynamic range that can be higher than conventional cameras,

- Smear-free images of fast moving objects,

- Processing efficiency: line scanning eliminates the frame overlaps required to build a seamless image, particularly in high-speed, high-resolution applications.

\subsection{Two-Type Panoramic Street Images}

When the line scan sensor moves, instead of objects, perpendicularly to the scanning line, line scan imaging is also realized and the same benefits can be received. Line scan imaging system mounted on a measurement vehicle exploits its abilities, when it runs at high speed in the outdoor environments. By using a line scan camera, two-dimensional and seamless images are easily acquired as shown in Figure 6. On the other hand, a laser range sensor generates three-dimensional and seamless images, shown in Figure 7.

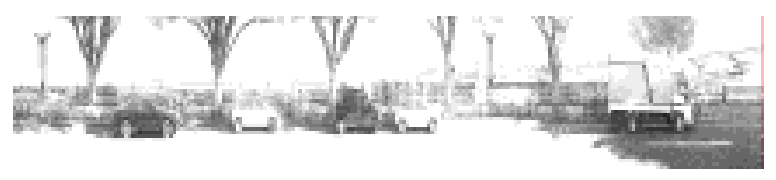

Figure 6 Panoramic street image using a line scan camera when it scans vertically

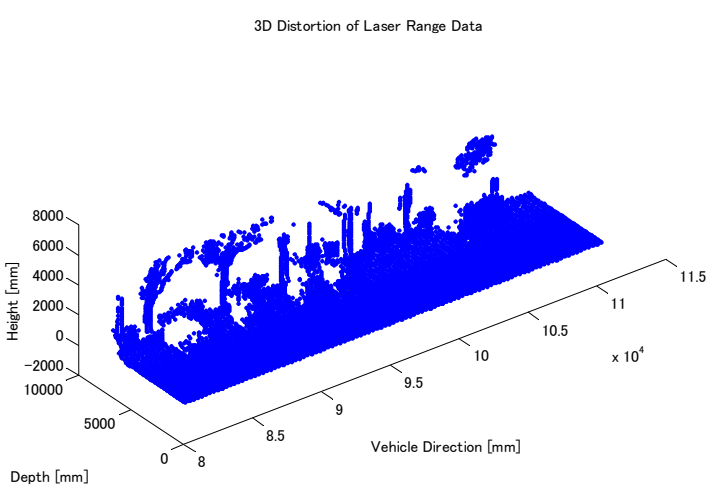

Figure 7 Panoramic street range image using a

laser range sensor when it scans vertically
Those seamless images provide original image data for modeling of buildings in urban cities and simulating cityscapes. The latest traffic navigation systems, such as car-navigation systems and driving simulators, need the details of geometric and photometric information about geographic features.

Those images include much and useful information for our everyday life, such as:

- Barrier-free facilities, such as steps between sidewalks and streets, that must be monitored on the way to an aging society,

- Road-use real situation, like street-parking vehicles and derelict bulky garbage,

- Road-traffic facilities, installed in the road traffic environments and public spaces, including traffic signs and road-side trees.

This paper is focused on the detection of street-parking vehicles, using the two-type panoramic street images.

\section{Detection Method Using Scanning Laser Range Sensor}

\subsection{Three-Dimensional Restoration}

The laser range sensor is mounted on the vehicle, and scan vertically and perpendicularly to the moving direction. Panoramic street images can be acquired.

The data acquired by the line-scan laser-range sensor are restored in the three dimension, by coordinate transformations among the three coordinate systems: (1) the sensor coordinate system, fixed on the sensor itself, ordinarily using the polar coordinate system with its origin at the laser detector, (2) the vehicle coordinate system, whose origin is the center of vehicle's mass and (3) the global coordinate system, fixed on the road and geostationary.

Results of three-dimesional restoration of the range data is shown in Figure 7. Occlusion occurs by objects near the laser sensor, and another object back the nearer objects from the laser sensor cannot be measured. Using laser is active sensing, on the other hand, a camera makes passive sensing. The laser range sensor is more robust than the camera, in the outdoor environments.

\subsection{Algorithm Using Laser Range Sensor}

Our proposed algorithm for detecting street-parking vehicles repeats the processing at each scan. This processing is consisted of the following steps.

(1) Compensating the decline of the sensor around 
the direction of the vehicle's movement: if no compensation of the sensor's decline, the road surface restored in the three dimension, is not horizontal, and affected by the vehicle's vibration. Figure 8 indicates the effect before and after compensation.

(2) Eliminating the range data consisting of the road surface: the range data of the road surface are assumed to have absolute value of height (the z-value) less than 100 millimeter.

(3) Eliminating the range data indicating limitations of measurement: for instance, in the experiments, the laser sensor can measure distance within 8191 meter, thus, the limitation is 8191 meter. Figure 7 shows the data after eliminating the limitations. Limitations form the arc at the center of the sensor.

(4) Extracting the range data with height less than 3000 millimeter: the height of usual vehicles is probably less than 3000 millimeter.

(5) Clustering the data indicating the vehicles side: if a street-parking vehicle is captured at the scan, a vertical cluster of range points is formed near the laser detector.

(6) Counting the appearance of clusters indicating the vehicles side: by tracking clusters from appearance until disappearance, street-parking vehicles can be counted.

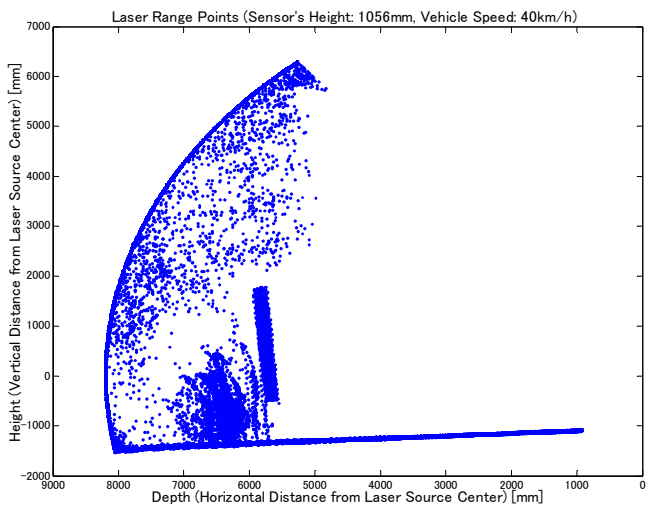

(a)

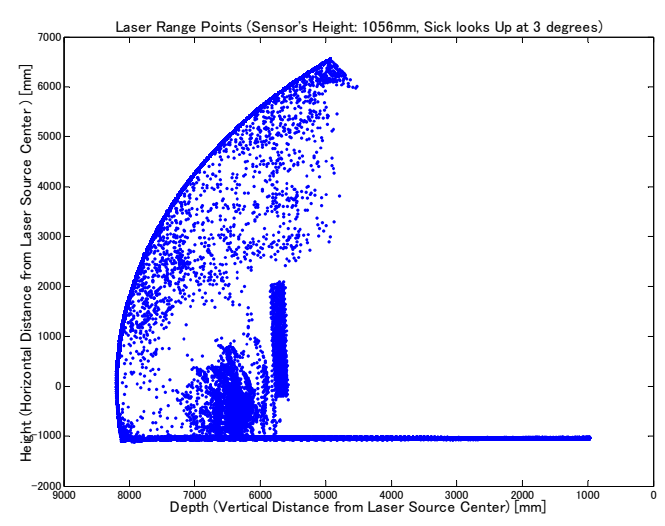

(b)

Figure 8 Range data before (a) and after (b) compensation of the declining road surface

\subsection{Experiments}

The laser range sensor was mounted on the measurement vehicle, and scanned vertically, as shown in Figure 9. Among 41 vehicles, 38 vehicles were be counted correctly, and 3 vehicles were be counted twice erroneously. The detection rate was $86 \%(38 / 44)$.
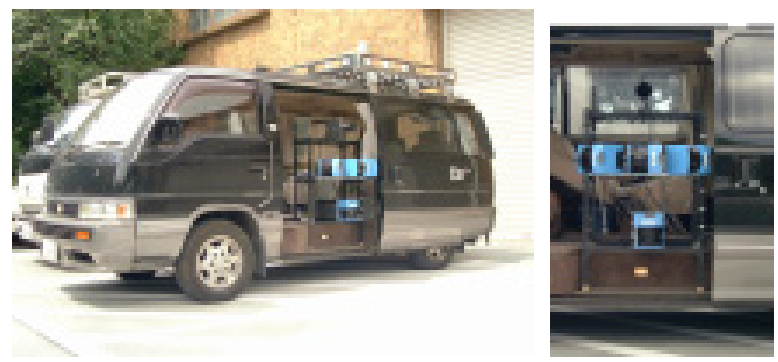

Figure 9 The line scan camera and the laser range sensors mounted on our measurement vehicle

\section{Extraction of Street-Parking Vehicle Images from Panoramic Street Image}

Line-scan imaging, synchronizing two-type line-scan sensors, the line-scan camera and the scanning laser range sensor, can simultaneously acquire geometric and photometric information. Not only counting street-parking vehicles from geometric information, but also specifying vehicles type from photometric information, may be realized.

The line-scan camera can capture a vehicle image, in the period between the appearance and disappearance of the vehicle in the laser-range data. Figure 10shows the results of extraction of vehicles from panoramic street images obtained by the line-scan camera. 


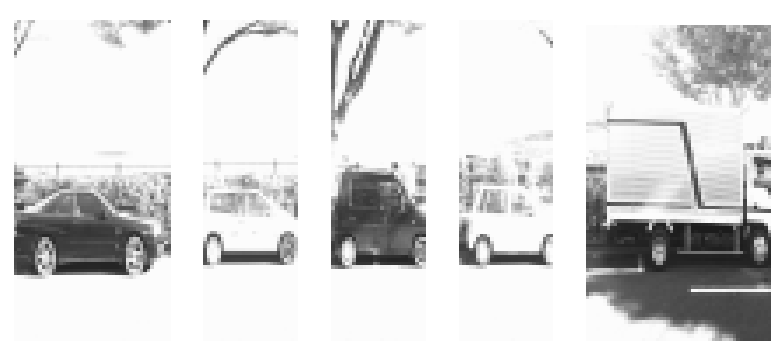

Figure 10 Extracted vehicles images

\section{Conclusion}

The detection method based on EPI analysis was proposed. Its detection rates are more than $65 \%$ as the experimental results.

We introduced the two-type panoramic street images. The panoramic street images were obtained by vertically scanning line-scan camera and laser range sensor. They include much and useful information for our daily life.

The other detection method, based on three-dimensional restoration of range data, achieved higher detection rate, more than $80 \%$. In addition, this method has been improved from the older method.

A laser sensor can measure the distance directly and robustly in the outdoor environment. However, an image sensor like a camera has much more information from data.

Thus, we will fuse an image sensor and a laser sensor, and improve the whole system. After extracting vehicles images from the panoramic street image, vehicle classification will be the next task.

\section{References}

(1) S. Ono, et al., "Parking-Vehicle Detection System By Using Laser Range Sensor Mounted on a Probe Car", Proc. of Intelligent Vehicle Symposium (IV'02), Poster Session 2, 2002.

(2) R. C. Bolles, et al., "Epipolar-Plane Image Analysis: An Approach to Determining Structure from Motion",

International Journal of Compute Vision, vol.1, pp.7-55, 1987.

(3) J. Canny, "A Computation Approach to Edge Detection", IEEE Trans. on Pattern Analysis and Machine Intelligence, 8(6), pp.679-698, 1986.

(4) P. V. C. Hough, "Machine Analysis of Bubble Chamber Pictures", Int. Conf. on High Energy Accelerators and Instrumentation, CERN, 1959.

Figure 11 Panoramic street image using a line-scan camera which scans vertically 